\title{
The statistical gauge in geometrical verification
}

Part I. Field of probability of the presence of matter

\author{
Jean Maihle $^{\mathrm{a}}$, Jean Marc Linares ${ }^{\mathrm{b}, *}$, Jean Michel Sprauel ${ }^{\mathrm{b}}$ \\ ${ }^{\mathrm{a}} \mathrm{EA}(M S)^{2}$, Aix Marseille Universities, Avenue Gaston Berger, F13625 Aix en Provence, France \\ ${ }^{\mathrm{b}}$ ISM/GIBO, UMR 6233, Aix Marseille Universities, Avenue Gaston Berger, F13625 Aix en Provence, France
}

\begin{abstract}
a b s t r a c t
Currently, most measurement and geometrical verification processes are based on the characterization of distances or angles between geometrical elements. In a previous paper, a method was developed for the verification of ISO specifications using the statistical information included in a set of points. This method is based on the development of measurement sequences and hence a sequence of geometrical constructions. The main problem in this verification process is to propagate uncertainties. With a correct expression of a virtual gauge, it is possible to avoid this propagation; indeed by using virtual gauge manipulation, initial best-fitted surfaces are never touched. The aim of this paper is to present a new approach using this statistical information to describe the measured part and develop a virtual gauge system for verification.

This will be done in two parts. The first part will focus on the statistical modelling of the measured surface; the second part will describe a verification method based on a virtual, i.e. numerical, gauge.
\end{abstract}

Keywords: Measurement Virtual gauge

Interference probability map Uncertainty propagation

\section{Introduction}

In a global industrial context, companies have to cope with stiff competition not only concerning costs, but also in rapidity as well as product quality. Moreover, in the past few years, companies have increasingly turned to outsourcing on an international level. Thus, mastering the assembly and lifespan of a mechanical system has become more and more complex. One of the most important parameters for this kind of problem is the inevitable geometric variation of a part when compared with its geometric specifications. These dispersions are due to the natural variability of the manufacturing process. At the present time, ISO geometric standards provide efficient concepts to specify parts. Thanks to current standards, variations can be limited thus respecting functional requirements. These specifications determine a geometric zone in which all real surfaces of the part are acceptable. Standards also provide methods which describe procedures for verifying these specifications. However, with the progress made in manufacturing processes and increasing demands for quality, tolerance intervals in geometric specifications are continually shrinking. Tolerance intervals are becoming near equals to the possibilities of the means of measurement. This situation has brought about an increase in the

Corresponding author at: Université de la Méditerranée/IUT d'Aix en Provence Av. Gaston Berger, F13625 Aix en Provence Cedex, France. Tel.: +33 4429390 96; fax: +33442939070

E-mail address: jean-marc.linares@univmed.fr (J.M. Linares). uncertainties of measurement. To cut back on verification uncertainties, two methods are possible: improving the quality of the measurement process or the part verification process.

Studies done on improving measurement processes have enabled researchers to create efficient methods of calibration and to compensate for geometric errors stemming from the means of measurement. But using these methods does not completely diminish measurement uncertainties. They do not take into account instantaneous random deviations or a form defect which cannot be compensated for. New technologies, whether optical or mechanical, can noticeably increase the rapidity in acquiring a measurement. Increasing the number of points measured allows us to use more and more complex and more and more accurate surface featuring techniques. Added to the new generations of three-dimensional modellers, these techniques open up new possibilities such as:

- best-fitting complex shapes,

- using statistical tools like Bayesian inference, and

- accurately estimating tangent surfaces.

In addition to raw measurement uncertainties, the geometrical verification process amplifies the error bars of modelled surfaces. Nowadays, the typical method of geometric verification uses vectorial calculation. It is based on distance determinations: point/point, point/line, and point/plane. The disadvantage of this kind of calculation is the fact that uncertainties can be generated while creating geometrical constructions. With current calculation capabilities, it is possible to make calculations which are less penalising 


\section{Nomenclature}

G vector representing a point of ${ }^{-}$

$a_{i} \quad i$ th coordinate of the vector if

$C_{x}, C_{y}, C_{z}$ localization parameters of the random vector $\mathbb{F}^{\mathbb{E}}$

$d \quad$ distance function

$E_{1}, E_{2}, E_{3}$ basis vectors of a coordinate system

$E$ first order statistical moment, i.e. mean value

$f \quad$ probability density function (PDF)

$g \quad$ any function

$g_{i} \quad i$ th value of the function $\mathrm{g}$ in the case of multidimen-

sional function

$\mathrm{H} \quad$ projected point

$J \quad$ Jacobian operator

$k_{i} \quad$ order of the statistical moment

$\mathrm{M}_{i} \quad$ measured point $i$

$n$ parameter number

Nbp number of measured point

$\operatorname{prob}(\overrightarrow{\mathrm{OM}})$ probability of a point $\mathrm{M}$ to be inside the matter

$p_{a} \quad$ level of risk of a SMB

$\mathrm{F}$ random vector representing mean estimated geo-

metric parameters

$P_{i} \quad i$ th coordinate of the random vector $\bar{F}$

$q(\mathrm{M}) \quad$ orthogonal projection of a point $\mathrm{M}$

$r, s, t$, curvilinear coordinate

$r_{0}, s_{0}, y_{0}, 0,0$ fixed curvilinear coordinate representing a given point of a surface

$R \quad$ radius

$\xi_{\bar{p}, \mathrm{x}}, \xi_{\mathrm{p}, \mathrm{y}}, \bar{\xi}_{\mathrm{p}, \mathrm{z}}$ parametric equation of the surface $S$, respectively, projected onto $x, y, z$

E orientation vector of the random vector $F$

$v_{\mathrm{x}}, v_{\mathrm{y}}, v_{\mathrm{z}}$ orientation parameter coordinates of $\mathrm{E}$

\section{Greek letters}

c, , w parameters of a plane with small displacement screw model

w likelihood function

standard deviation

2 variance

$\partial \mathrm{g}_{\mathrm{i}} \quad$ partial derivative of the function $g_{i}$ in respect with

$P_{j}$

v vector space

by replacing the usual geometrical construction with a virtual gauge.

This paper proposes a verification process using virtual calibration with a statistical criterion. The goal is to provide the metrologist with an indication of the risk he is taking when he claims that a part is geometrically standard. The novelty of this method is to include measurement uncertainties in the verification process. This indication must also make it possible to question the measurement strategy used, the sampling strategy and the number of points probed.

This paper is divided into two distinct parts. The first part will put forward a statistical modelling method of real surfaces. This model is presented in the form of a field of probability of the presence of matter (FPPM). It enables us to take any point in space and calculate the probability of its being within the boundaries of the object in question.

The second part deals with a method of geometrical verification of a part using virtual gauges. Virtual gauges will be modelled by a metric tensor and, thanks to the FPPM, will enable

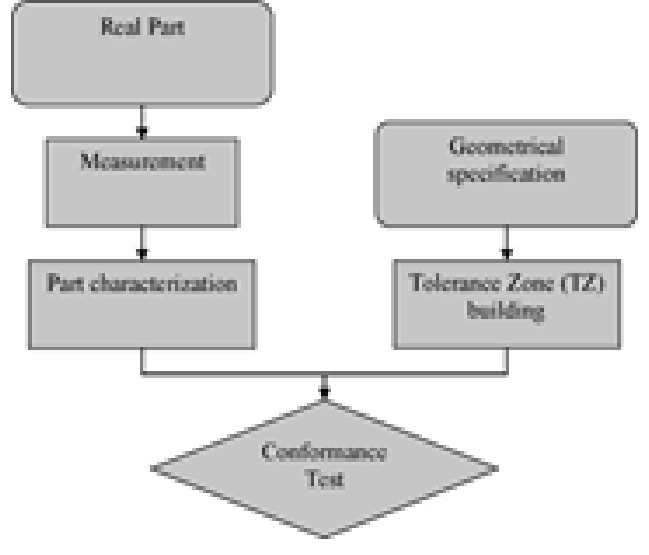

Fig. 1. Verification section.

us to have a geometrical check on the product using a statistical criterion.

\section{State of the art}

Geometrical verification using coordinate measuring machines (CMM) can be summed up in four different actions (Fig. 1):

- Measuring the real part.

- Determining the features of the real surfaces of the part.

- Interpreting geometrical specifications.

- Testing conformance.

A great variety of research work has been carried out on the measurement of geometrical quantities as well as on verification. These studies cover many fields which can be organized into four groups: best-fitting methods, uncertainty calculations, tolerance zone descriptions, and conformance testing. Fig. 2 proposes different channels of research for each group.

In the first part of this paper, we will present different studies done in the fields concerning how to determine the features of measured surfaces as well as fields concerning how to estimate uncertainty.

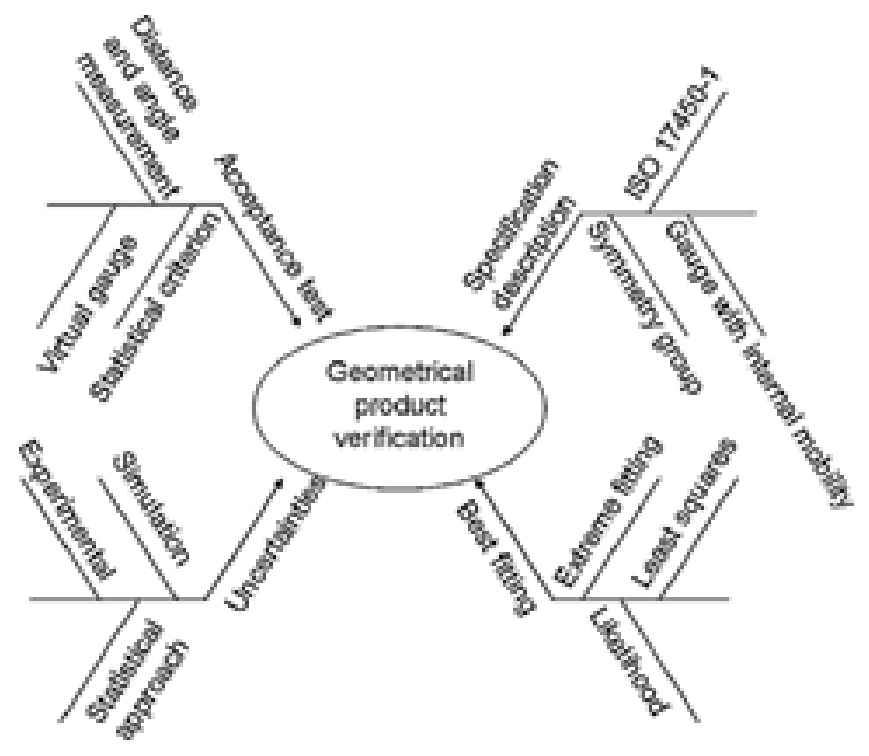

Fig. 2. Fields of geometrical verification with a CMM. 


\subsection{Best-fitting}

The data supplied by CMM is given in the form of point coordinates. To estimate the surface of the real part, best-fitting must be carried out. Best-fitting is the operation in which a given feature representing the real surface is associated with a set of measured points. This association is completed by optimizing a criterion like least squares, infinite norm or likelihood functions. Since the beginning of CMM use, research work has been done on numerous best- ttin methods [1-3]. In fact, the minimized function is never linear, but to simplify the optimization problem, the objective function is often linearized. A method based on a likelihood function proposes a non-linear optimization association [4]. Nevertheless, it is well known in measurement that the sampling of the real surface and hence best-fitting, imply that the estimation of the surface is provided with uncertainties.

\subsection{Uncertainties}

Research on uncertainty calculation for CMM can be organized into three classes:

- Experimental measurement of gauge blocks: Different artefacts, user-defined parts or geometrical gauges can be used to calibrate and determine uncertainty in CMM $[5,6]$.

- Simulations of the measurement process: The Monte Carlo Method is commonly used for simulating complex measurement processes. It has thus been used as an alternative to treat the complex measurement of dimensions and to determine the probability distribution density of measured quantities numerically [7-9].

- The statistical approach: Least squares and extreme fits are used in CMM and a variety of researchers have developed several numerical algorithms for three-dimensional data analysis. A statistical relationship method has been developed to determine the validity of the geometric model [10]. The same research group created a method to estimate the uncertainty of measurement on envelope surfaces [11]. Evaluating uncertainties in the vectorial tolerances using the least squares method has been proposed. The rotation and translation vectors of the surface were combined to form a homogeneous coordinate transformation matrix which, however, was approximated to the first order expansion [12]. A number of projects related to the study of a set of measured points have been carried out. Methods have been suggested to evaluate geometric errors obtained on parts when the manufacturing process is known [13,14]. Finally, research has been done on estimating the measurement uncertainty of small circular features measured by CMM [15].

\section{Modelling surfaces using random vectors}

Studying the bibliography, we can assert that classic methods of geometrical verification of products do not allow us to claim without a doubt that a product complies. Current research does not directly take into account uncertainties in the verification of geometrical specifications. It is limited, first of all, to determining the magnitude of the measured feature; then, calculations of measurement uncertainties are made separately. To compensate for this problem, we are going to present surface modelling based on the notion of random vectors. In this way we will be able to determine the uncertainty of the complete surface. This method will be presented in this chapter and used in the rest of the document to obtain a statistical index of the compliance of a product to a system of geometrical specifications.

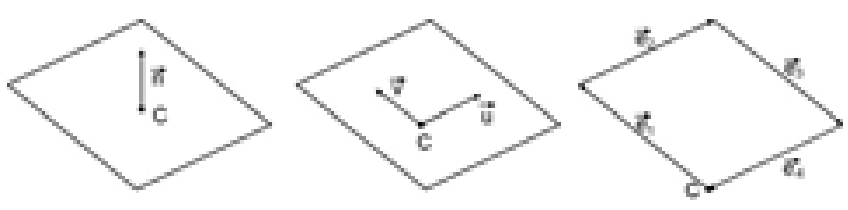

Fig. 3. Point/vector descriptions of a plane.

\subsection{The "point, vector, parameter" model}

All geometric surfaces, which can be described in an equation can be represented with a set of point/vectors and intrinsic parameters. This assembly of a point and one or more vectors allows locating and orienting the surface. The intrinsic parameters enable us to construct the surface. There are several solutions in modelling. They are based on sets of points and/or vectors representing the same surface (Fig. 3).

However, statistical descriptions of a surface are more efficient if the number of parameters is reduced to the bare minimum (principle of parsimony). Thus, the random vector modelling method is the one proposed in the table of "point/vector/scalar" modelling (Fig. 4).

In the rest of the document, all the scalars in this modelling (point coordinates, coordinates of the vector(s) and parameters intrinsic to the model) will make up a vector shown as $\mathbf{F}$. To illustrate this approach, let's take the case of a cylinder (Fig. 5). The random vector of the cylinder is made up of a point, a vector and a radius.

The random vector $\mathbf{F}$ used here is derived from a minimal random vector which was used in best-fitting.

\subsection{Statistical best-fitting: vector expectation and the covariance matrix}

The real surface to be estimated is made up of an infinite number of points. Statistically, the infinite number of points of the surface produces a population to be characterized. The set of points evaluated can thus be considered as a statistical sampling of this population. Modelling by random vectors consists in estimating the first and second statistical moment of this vector, in other words, the mathematical expectation and the set of covariances in vector P.

\subsubsection{The random vector}

A random vector $\bar{F}$ of $n$ dimensions belonging to $<^{\mathrm{n}}$ is the vector $\left[\mathrm{P}_{1}, \mathrm{P}_{2}, \ldots, \mathrm{P}_{\mathrm{n}}\right]^{\mathrm{T}}$. Its components. $P_{i}$, are random variables. The random vector components are assumed to be continuous. The

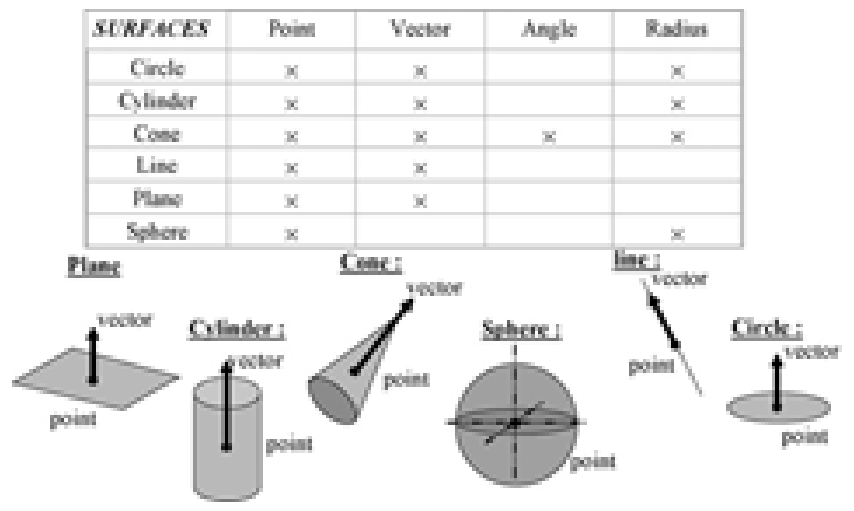

Fig. 4. Point/vector/scalar modelling. 


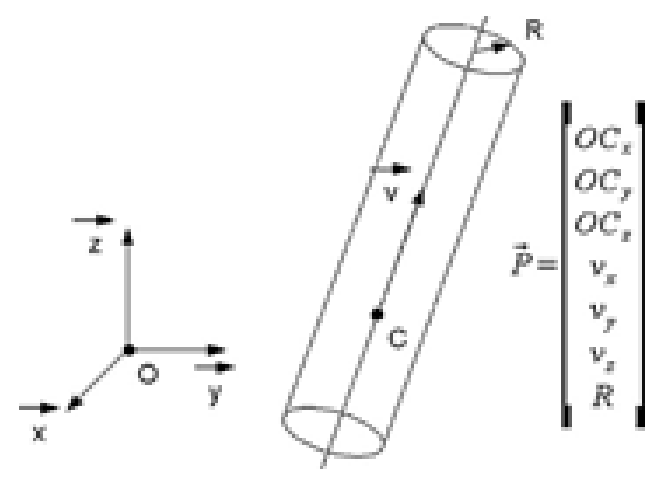

Fig. 5. Random vector of a cylinder

probability density function $f$ is defined as:

$\left.f:<^{\mathrm{n}} \rightarrow\right] 0,1[$

$\mathrm{P} \rightarrow \mathrm{f}(\mathrm{F})$

The probability that $\bar{F}$ is found within a space, ${ }^{\prime}$, is given by the function $f$ defined as follows:

ZZ Z

$\ldots \quad f\left(a_{i}\right) d a_{i}=\operatorname{prob}\left(\bar{F}_{\in}{ }^{\cdots}\right)$

$\cdots$

The standard properties defined in one-dimensional statistics remain valid:

- Probability density $f$ has values in $<+$ ZZ

- The value of the repartition function on $<^{\mathrm{n}}$ is: $\begin{array}{ll}Z Z & R \\ & f\left(a_{i}\right) d a_{i}=\end{array}$ 1

We assume that the components of random vector are continuous with a probability density $\mathrm{f}$ which admits statistical momenps. These moments are defined by the following integral: $\mathrm{m}_{\mathrm{k}_{\mathrm{i}}}=\ldots \mathrm{a}_{\mathrm{i}}^{\mathrm{k}_{\mathrm{i}}} \mathrm{f}\left(\mathrm{a}_{\mathrm{i}}\right) \mathrm{du}_{\mathrm{i}}$ with: $k_{i} \in N$ designating the moment of order $k$ of the variable $i$ of vector $\bar{F}$.

If the random components $\bar{P}$ of the vector contain a mathematicap expectation, the centered moments can be calculated: $\mathrm{k}=\quad \ldots\left[\mathrm{a}_{\mathrm{i}}-\mathrm{E}\left(\mathrm{a}_{\mathrm{i}}\right)\right]^{\mathrm{ki}} \mathrm{f}\left(\mathrm{a}_{\mathrm{i}}\right) \mathrm{da}_{\mathrm{i}}$.

The concept of a covariance matrix can be very useful for representing the second order moments.

\subsubsection{The first two moments of the random vector}

The preceding section defined the different moments of the random vector via the hypothesis of existence. Deriving from the basic concepts of multi-dimensional statistics, the expectation vector and the variance covariance matrix of a random vector can be defined.

When $\bar{F}$, random vector of $n$ dimensions and components $\left[\mathrm{P}_{1}, \mathrm{P}_{2}, \ldots, \mathrm{P}_{\mathrm{n}}\right]^{\mathrm{T}}$, has first order moments, its expectation vector is defined as follows:

\begin{tabular}{c|c}
$\mathrm{E}(\boldsymbol{\mathrm { F }})$ & $\mathrm{E}\left(\mathrm{P}_{1}\right)$ \\
$\mathrm{E}\left(\mathrm{P}_{2}\right)$ \\
$\cdot$ \\
$\cdot$ \\
$\mathrm{E}\left(\mathrm{P}_{\mathrm{n}}\right)$
\end{tabular}

Given that $\overline{\mathrm{P}}$, a random vector of $n$ dimensions and components $\left[\mathrm{P}_{1}, \mathrm{P}_{2}, \ldots, \mathrm{P}_{\mathrm{n}}\right]^{\mathrm{T}}$ which admits second order moments, and thus

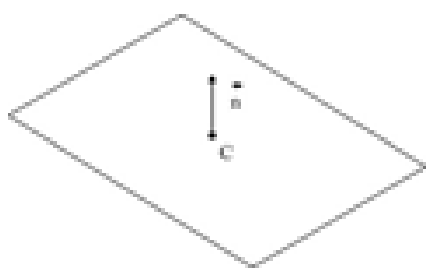

Fig. 6. Random vector of a plane (point and orientation vector)

first order phoments, its variance covariance matrix is defined as:

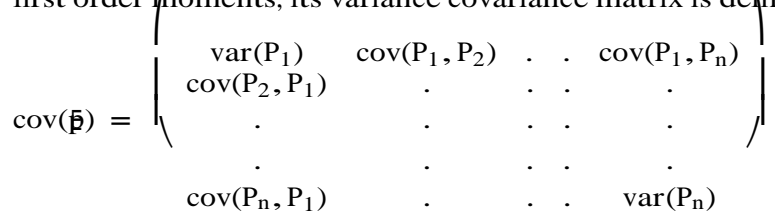

The variance covariance matrix is a square matrix of $n \times n$ dimensions.

For example, in the case of a plane defined by a point $\mathrm{C}$ and a normal vector (Fig. 6), the expectation vector and the variance covariance matrix are:

For the first statistical moment:

$\mathrm{E} \mathrm{P}={ }^{1} \mathrm{C}_{\mathrm{x}} \quad \mathrm{C}_{\mathrm{y}} \mathrm{C}_{\mathrm{z}} \quad v_{\mathrm{x}} \quad v_{\mathrm{y}} \quad v_{\mathrm{z}}$

$\overline{\text { For the second statistical moment: }}$

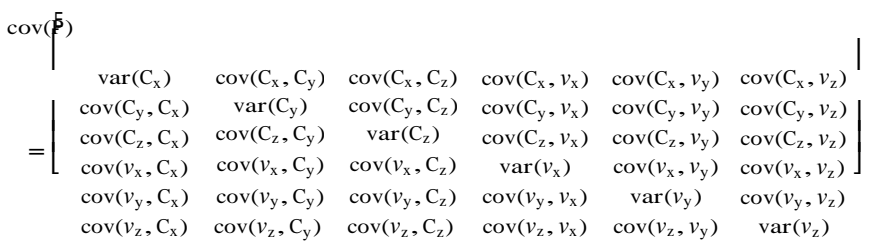

\subsubsection{Best-fitting and estimating uncertainties}

The first step in best-fitting is choosing a model to describe the measured surface. The model is an equation of the surface. It must enable us to make a best fit based on a statistical criterion and to express the residues of the best fit. The calculation is carried out by expressing the distance between probed points and the model to best fit. Models used in this paper are based on the description "point, vector(s), parameter(s)" described in Section 3.1 used as a basis for the random vector. These models are expressed in parametric form:

$\mathrm{\xi}_{\overline{\mathrm{F}}}(\mathrm{u}, v)$

where $u$ and $v$ represent curvilinear coordinates of the surface. The distance between point $\mathrm{M}$ and the best-fit model is marked as $d$ and given by the function $d$ defined as:

$\mathrm{d}:<^{3} \rightarrow<$

$\mathrm{d}\left(\mathrm{M}_{\mathrm{j}}\right) \rightarrow \mathrm{d}_{\mathrm{j}}$

The second step is the choice of the best-fitting criterion. This criterion depends on the hypothesis taken on the distribution function of residuals [16] written as the series $f$.

The criterion used is maximum likelihood which gives an independent generic approach to function $f$. The goal is to maximize the likelihood function defined as:

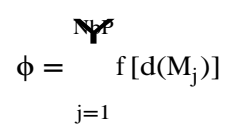

where NbP represents the number of data points. 
Function $\phi$ only presents one overall maximum for surfaces produced by our means of production. This property has been shown in previous work [17]. The condition to check is to solve the following system of equations:

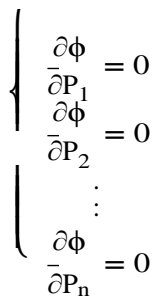

where $n$ represents the number of parameters.

In the case of a Gaussian probability density function, the problem can be expressed in the following way:

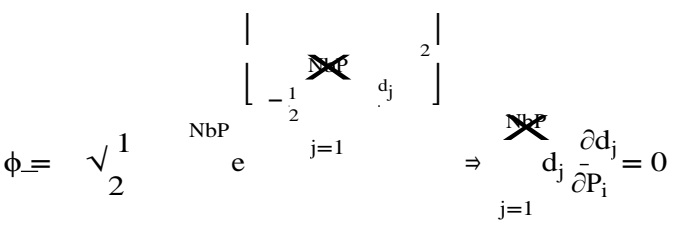

If the $n$ parameters, $P_{i}$, of the geometric element associated to the digitized surface were perfectly defined, the standard deviation could also be estimated in the same way:

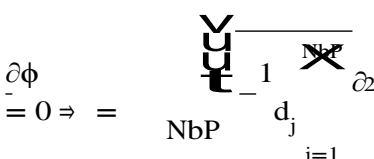

However, such estimator would lead to a biased evaluation of because a set of $n$ parameters $P_{i}$ has already been derived from the acquired data. Therefore, the standard deviation of the measurement has to be computed with the following expression, also called residue of the least squares optimization:

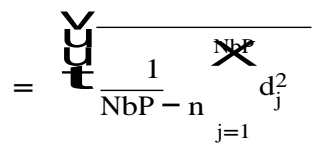

This deviation, , can be propagated to deduce the covariance matrix of the estimated parameters $P_{i}$, using classical differential expressions of the uncertainties. From the diagonal components of the covariance matrix, the error bars of $P_{i}$ are then easily calculated. since the statistical distribution of these random variables corresponds to a Fisher-Student law. Moreover, the covariance matrix is also useful to propagate the uncertainties of the fitted surfaces to any derived geometric element.

It has to be pointed out that the propagation process of the standard deviation requires a precise definition of the derivatives $\partial d_{j} / \partial P_{i}$. For that reason, a nonlinear algorithm, based on a Gauss-Newton type method, has been implemented to solve the optimization conditions. It does not require any coordinate transformation and is not sensitive to the selection of initial intrinsic parameters. It allows proceeding all the classical surfaces, i.e. lines, planes, circles, cylinders, cones and spheres.

Usually, the measurement uncertainties are assumed the same for all the digitized coordinates. Nevertheless, this hypothesis in not required to apply the maximum likelihood criterion. In fact, if the standard deviation, ${ }_{j}$, of each distance $d_{j}$ is well known, a weighted least squares criterion can be minimized to estimate the parameters of the fitted surface.

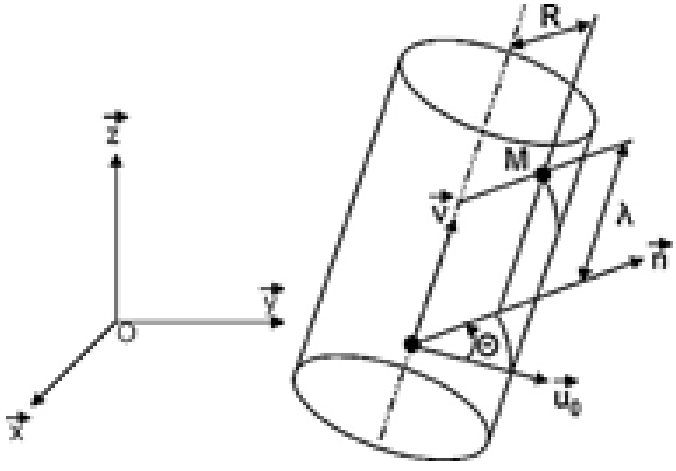

Fig. 7. Parametric formulation of a cylinder.

\section{Field of probability of the presence of matter}

\subsection{Objective and description of the method}

The next sections of the paper will define the probability that the real surface is, totally or partially included in the specified tolerance zone. For that purpose, the probability, written prob $\overrightarrow{\mathrm{OM}}$, that any point $\mathrm{M}$ of the $3 \mathrm{D}$ space is within the matter side of the real surface will be expressed. This model is called Field of probability of presence of the matter (FPPM). At the current step of the method, the random vector of the surface has already been estimated. It represents the mean value and the set of variance/covariances of the geometrical parameters of the vector/point/dimension obtained during the best-fitting (described in Section 3). The expression of the FPPM is done in three steps. First. the distance between any point of the 3D space and the mean surface has to be calculated. Its standard deviation has then to be derived from the variance/covariances of the random vector. Hence a parametric formulation of the mean surface must be written for these two operations. Finally the value of the FPPM is obtained by a simple integration of the Gaussian law. In this part, a formulation enabling us to define the function prob $\overrightarrow{\overline{\mathrm{OM}}}$ will be presented.

\subsection{Parametric formulation of mean estimated surface}

The first step in calculating probability prob $\overrightarrow{\mathrm{OM}}$ is to find a parametric form for the mean estimated surface. This formulation will enable us to develop the estimated mean surface from the first statistical moment of random vector $\overline{\mathrm{F}}$. In some cases, a Cartesian formulation may appear more appropriate as in the case of a plane. However, such logic does not allow for a generic approach to the problem of verification.

The idea put forward here is to develop a parametric equation $\bar{S}_{\bar{E}}(r, s)$ representing the complete associated surface. It will be defined with the random vector $\overline{\mathrm{P}}$, where $r$ and $s$ are the curvilinear coordinates of the surface. Fig. 7 shows an example of a cylindrical associated surface.

The next section will show how to propagate the uncertainties of a random vector to a derived integral element.

\subsection{Propagating uncertainties}

\subsubsection{The principle of propagation}

The previous sections explained how to evaluate the uncertainties of a random vector by linearly propagating the variance of a one-dimensional random variable. The uncertainty propagation of a random vector of any dimensions toward another vector of any dimensions will be now presented. 
Given any function $g$ of $<^{\mathrm{n}}$ in $<$, the variance of the value of $g$ for a random vector $\overline{\mathrm{F}}$ of $n$ dimensions is expressed as:

$\operatorname{var}[g(\bar{P})]=\underset{j=1 \quad i=1}{\boldsymbol{X} \times} \frac{\partial g(\bar{P})}{\partial P_{i}} \frac{\partial g\left(\bar{F}^{\mathbf{P}}\right)}{\partial P_{j}} \operatorname{cov}\left(\mathrm{P}_{i}, P_{j}\right)$

In the case of a function $g$ of $<^{\mathrm{n}}$ in $<^{\mathrm{m}}$, the covariance of the random vector $\mathrm{g}(\overline{\mathrm{F}})$ is expressed as:

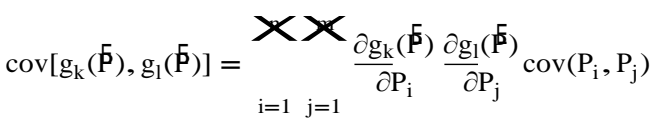

Using the Jacobian matrix of function $g$, the propagation of uncertainties can be written in a matrix form as follows:

$\operatorname{cov}[\mathrm{g}(\overline{\mathrm{P}})]=\mathrm{J}_{\mathrm{g} / \mathrm{F}} \cdot \operatorname{cov}(\mathrm{g}) \cdot \mathrm{J}_{\mathrm{E} / \mathrm{F}}^{\mathrm{T}}$

$\mathrm{J}_{\mathrm{g} / \mathrm{F}}=\left|\begin{array}{ccccc}\frac{\partial \mathrm{g}_{1}}{\partial \mathrm{P}_{1}} & \frac{\partial \mathrm{g}_{2}(\overline{\mathrm{F}})}{\partial \mathrm{P}_{1}} & \ldots & \ldots & \frac{\partial \mathrm{g}_{\mathrm{m}}(\overline{\mathrm{F}})}{\partial \mathrm{P}_{1}} \\ \frac{\partial \mathrm{g}_{1}(\overline{\mathrm{F}})}{\partial \mathrm{P}_{2}} & \ldots & \ldots & \ldots & \ldots \\ \ldots & \ldots & \ldots & \ldots & \ldots \\ \frac{\partial \mathrm{g}_{1}(\overline{\mathrm{P}})}{\partial \mathrm{P}_{\mathrm{n}}} & \ldots & \ldots & \ldots & \frac{\partial \mathrm{g}_{\mathrm{m}}(\overline{\mathrm{F}})}{\partial \mathrm{P}_{\mathrm{n}}}\end{array}\right|$

where $\frac{\partial \mathrm{g}_{i}(\bar{F})}{\partial \mathrm{P}_{\mathrm{j}}}$ represent the $i$ th derivative of the function in respect to $j$ th coordinate of $\mathbf{F}$.

In Section 4.2, the estimated mean surface of a real feature was represented by the parametric equations $\overrightarrow{\overline{\mathrm{S}_{\mathrm{E}}}}(\mathrm{r}, \mathrm{s})$. The uncertainties of the random vector $P$ will be propagated, through this equation, to any point of the surface. For a fixed set $r$ and $s$, shown as $u_{0}$ and $v_{0}$, the coordinates of point $\mathrm{H}$ are thus obtained within the mean surface: $\overrightarrow{\mathrm{OH}}=\overrightarrow{\mathrm{S}_{\overline{\mathrm{p}}}}\left(\mathrm{r}_{0}, \mathrm{~s}_{0}\right)$. The aim is to determine the threedimensional variance of this same point $\mathrm{H}$. It will be calculated from the covariance matrix of the random vector $\overrightarrow{\mathrm{OH}}$. Using the method defined before. This propagation is achieved in the following way:

$\operatorname{cov} \overrightarrow{\mathrm{OH}}=\operatorname{cov}\left[\mathrm{F}_{\overline{\mathrm{p}}}\left(\mathrm{r}_{0}, \mathrm{~s}_{0}\right)\right]=\mathrm{J}_{\bar{E} / \mathbb{E}} \cdot \operatorname{cov}(\overline{\mathrm{F}}) \cdot \mathrm{J}_{\bar{E} / \bar{E}}^{\mathrm{T}}$

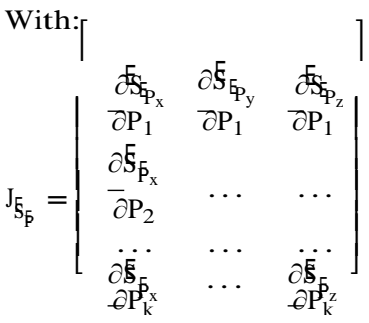

In some cases, the parametric formulation of the mean surface requires using a local coordinate system. The make-up of the coordinate system must be formulated and the uncertainties of the initial random vector must be propagated to the reference point. The propagation formula is found to be:

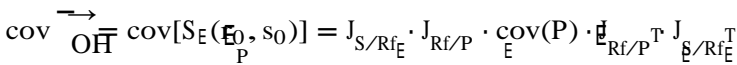

where $R f$ represents the local coordinate system, and $J_{5 / R f}$ represents the Jacobian matrix of the parametric equation in relation to the local coordinate system. The $J_{R f / F}$ is the Jacobian matrix of the construction of the local coordinate system in relation to the random vector $\bar{P}$.

The next sections present an application of uncertainty propagation in the case of a plane and a cylinder.

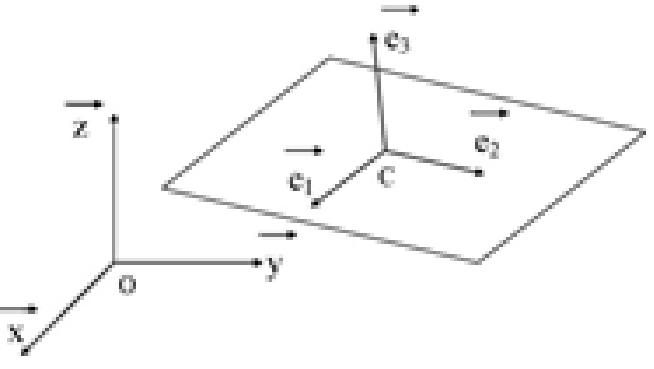

Fig. 8. Parametrizing a plane.

4.3.2. Uncertainty propagation in the case of a plane

1 The random vector $\mathrm{P}$ of a plane is $\overline{\mathrm{P}}=$ $\begin{array}{llllll}\mathrm{OC}_{\mathrm{x}} & \mathrm{OC}_{\mathrm{y}} & \mathrm{OC}_{\mathrm{z}} & v_{\mathrm{x}} & v_{\mathrm{y}} & v_{\mathrm{z}}\end{array}$. To clarify the parametric equation, a local coordinate system $\left(C, E_{1}, E_{2}, E_{3}\right)$ can be constructed:

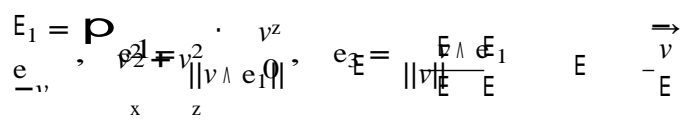

The Jacobian matrix $\mathrm{J}_{\mathrm{Rf} / \mathrm{F}}$ of the construction of the coordinate system can be derived from the expressions of the coordinates of these vectors. Parametrizing the mean surface of the plane is achieved in Fig. 8.

The parametric equation of the plane is:

$\xi_{\mathrm{p}}(\mathrm{r}, \mathrm{s})=\mathrm{r} \cdot \mathrm{E}_{1}+\mathrm{s} \cdot \mathrm{E}_{2}$

A fixed point $\mathrm{H}$ in the plane is expressed as:

$\overrightarrow{\mathrm{OH}}=\xi_{\overline{\mathrm{p}}}\left(\mathrm{r}_{0}, \mathrm{~s}_{0}\right)=\overrightarrow{\mathrm{OC}}+\mathrm{r}_{0} \cdot E_{1}+\mathrm{s}_{0} \cdot E_{2}$

The Jacobian matrix used to propagate uncertainties toward point $\mathrm{H}$ is:

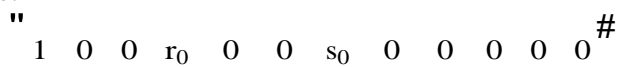

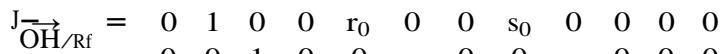

In conclusion, the three-dimensional variance of point $\mathrm{H}$ within the estimated mean plane is:

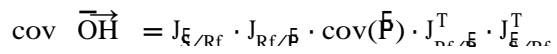

\subsubsection{Propagating uncertainty in the case of a cylinder}

1 The random vector $\mathrm{P}$ of a cylinder is $\mathrm{P}=$ $\begin{array}{llllllll}\mathrm{OC}_{\mathrm{x}} & \mathrm{OC}_{\mathrm{y}} & \mathrm{OC}_{\mathrm{z}} & v_{\mathrm{x}} & v_{\mathrm{y}} & v_{\mathrm{z}} & \mathrm{R} & \text {. To clarify the parametric }\end{array}$ equation, the local coordinate system $\operatorname{Rf}\left(C, E_{1}, E_{2}, E_{3}, 1\right)$ can be constructed. The unit, shown as " 1 " in reference frame, is used as a basis for the parameter representing the radius of the cylinder. The components of the local coordinate system vectors are defined as follows:

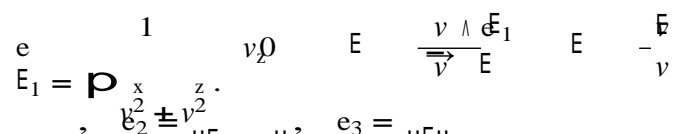

The Jacobiln' 'mat'ix of the 'cEnstruction of coordinate system $R f$, named $\mathrm{J}_{\mathrm{Rf} / \mathrm{F}}$, can be derived from these expressions. Parametrizing the mean surface of the cylinder is achieved in Fig. 9.The parametric equation of the surface is obtained:

$\overrightarrow{\mathrm{OH}}=\mathrm{S}_{\overline{\mathrm{F}}}()=,v+\mathrm{E} n() \mathrm{E}$

With

$\mathrm{E}()=\cos () \cdot \mathbf{u}+\mathrm{E} \sin () \cdot \mathrm{W} \quad \mathrm{E}$ 


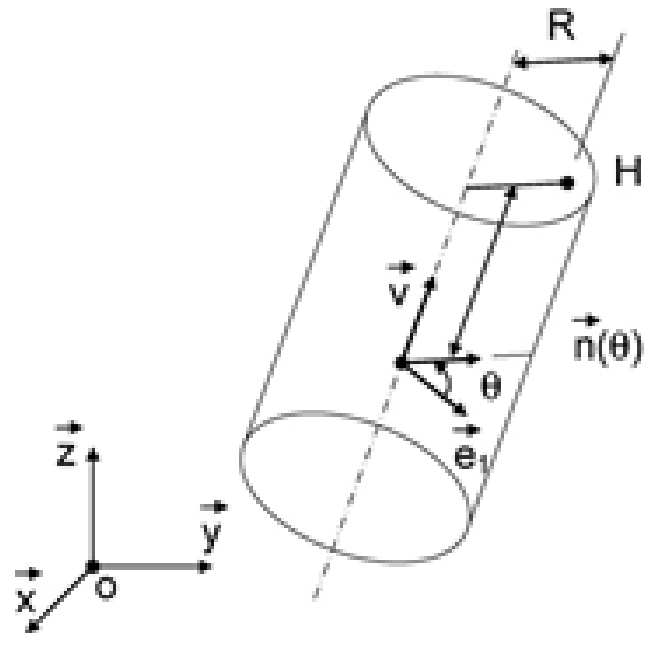

Fig. 9. Parametrizing a cylinder.

with being the axial parameter, i.e. the height in relation to center $C$ in the direction of vector $E v$, and the radial parameter. The Jacobian matrix used to propagate uncertainties toward point $\mathrm{H}$ is:

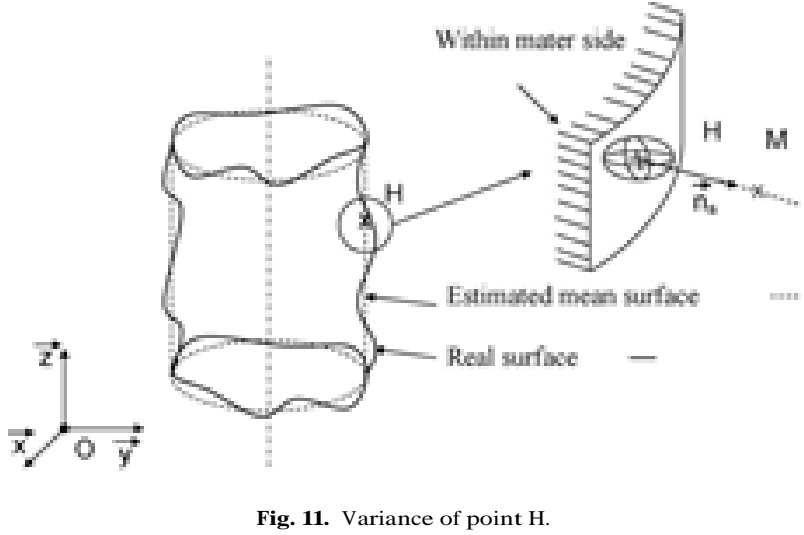

distribution of probability representing the position, or the coordinates, of point $\mathrm{H}$. As indicated in Fig. 11, point $\mathrm{H}=q(\mathrm{M})$ is the orthogonal projection on the mean surface of any point $M$ in a $3 \mathrm{D}$ space. To define the FPPM or the probability that M is part of matter, only the dispersion of point $\mathrm{H}$ in the normal direction to the surface is required (Fig. 11).

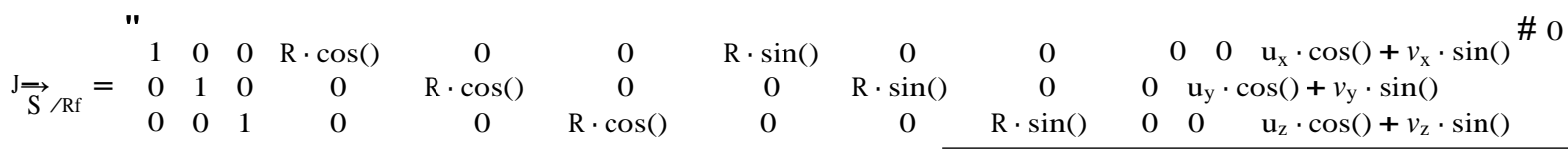

Finally, the three-dimensional variance of point $\mathrm{H}$ within the estimated mean cylinder is:

$\operatorname{cov} \overrightarrow{\mathrm{OH}}=\mathrm{J}_{\xi / R f} \cdot J_{\mathrm{Rf} / \bar{F}} \cdot \operatorname{cov}(\overline{\mathrm{F}}) \cdot \mathrm{J}_{\mathrm{Rf} / \overline{\mathrm{F}}}^{\mathrm{T}} \cdot \mathrm{J}_{\bar{\xi} / \mathrm{Rf}}^{\mathrm{T}}$

\subsection{Formulating the field of probability of the presence of matter (FPPM)}

The goal of our statistical modelling is to clarify a function prob $\overrightarrow{\mathrm{OM}}$ of $<^{3}$ in $<$ which, at any point $\mathrm{M}$ in space, associates its probability of being within matter. This calculation is carried out in four steps (Fig. 10).

In the above section, the result of propagating uncertainties of the random vector toward point $\mathrm{H}$ within the estimated mean surface was the three-dimensional variance of this point. In other words, propagating uncertainties allows us to characterize the $3 \mathrm{D}$

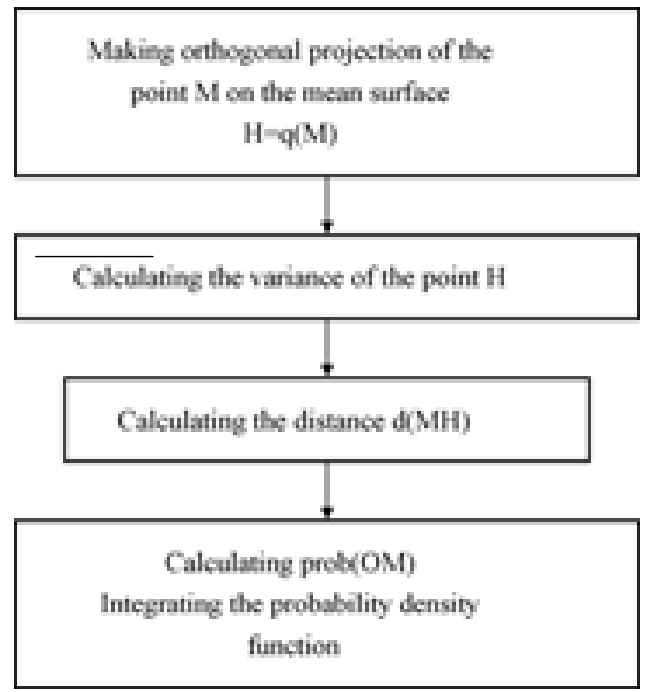

Fig. 10. The steps in calculating prob(M)
The dispersion of point $\mathrm{H}$ in the normal direction of the mean surface is obtained by projecting onto a unitary normal vector (shown as $\bar{E}_{S}$ in Fig. 11). The projection is demonstrated by the relation: $\underset{\mathrm{O}}{\stackrel{2}{\overrightarrow{\mathrm{Hn}}} \overrightarrow{\mathrm{S}}}=\mathrm{n}_{\mathrm{S}}^{\stackrel{\rightarrow}{\longrightarrow}} \cdot \operatorname{cov} \mathrm{O} \overline{\mathrm{H}} \cdot \mathrm{n}_{\mathrm{S}} \overrightarrow{.}$

To get the total local variance, adding the variance of the residues obtained in best-fitting (the statistical representation of the deviations due to the form defects and the random perturbations of the CMM) will suffice: ${ }_{\text {Total }}^{2}=\mathrm{n}_{\mathrm{S}}=\underset{. \mathrm{n}_{\mathrm{S}}}{2} \underset{\text { Residues }}{\stackrel{2}{2}}$

The standard deviation in the normal direction of the mean surface and the position of point $\mathrm{H}$ enables us to give a complete definition of the probability density function to integrate in order to calculate prob $\mathrm{OM}$. To carry out this operation, the integration bine has to be clarified. This line is defined by point $\mathrm{H}$ and direction $\mathrm{n}_{\mathrm{S}}$ (Fig. 12).

This representation makes it possible to visualize the bounds used in the integral: $[-\infty ; d(\mathrm{MH})]$. Our application will be restricted to surfaces of high quality. In this case, the probability density of the position of a point within an estimated mean surface has a Gaussian behaviour [16]. The following expression of FPPM is thus obtained:

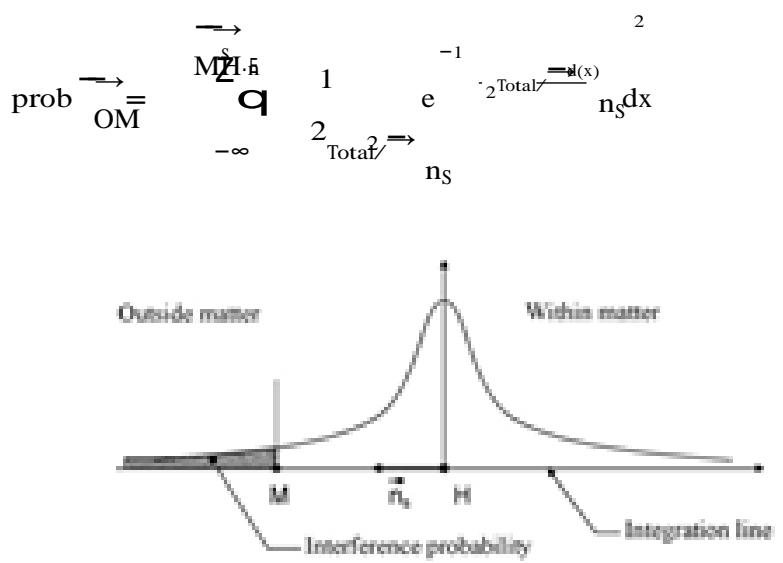

Fig. 12. Integration principle. 


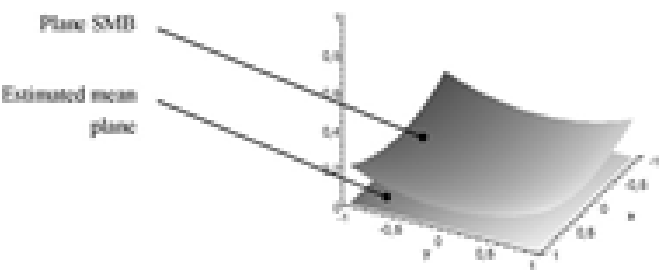

Fig. 15. SMB of a plane.

equation:

$\left(x-O C_{x}\right) n_{x}+\left(y-O C_{y}\right) n_{y}+\left(z-O C_{z}\right) n_{z}-k \overline{v a r(d(x, y, z))}=0$

The surface obtained is a paraboloid (Fig. 15) where sections parallel to the mean plane are elliptical. As the cylinder case, the distortion obtained is depending on the uncertainty of the orientation parameters of the mean estimated plane.

\section{Conclusion}

In this paper, a statistical approach of CMM measurements has been proposed to characterize any real surface.

In this approach, a geometric element is no longer defined by a perfect feature but is described by a FPPM. This field, based on the concept of random vectors, defines the probability of any point to stay inside the matter. It integrates the random perturbations of the CMM, the uncontrolled factors of the sampling strategy and the form defects of the measured surface.

This concept can be applied to any standard surface (plane, sphere, cylinder, cone, ...) and extended to models accounting for typical form errors thus increasing the significance of the best-fit method. This method has been tested with experiments on classical geometrical specifications [16]. The increase of the number of best-fit parameters to describe and fit the measured surfaces leads to a better characterization of the form defects and thus reduces considerably the uncertainties [18].

The second part of our work will focus to the implementation of a virtual gauge method integrating the field of probability of the presence of matter. It will permit checking the geometrical conformance of a real part defining the risk of acceptance or refusal of the product. It will automatically include all errors or random perturbations and avoid any intermediate geometrical construction which amplifies the initial measurement uncertainties.

\section{References}

[1] Bourdet P, Mathieu L, Lartigue C, Ballu A. The concept of small displacement torsor in metrology. Ser Adv Math Appl Sci 1996;40:110-22.

[2] Cox MG, Forbes AB, Flowers JL, Harris PM. Least square adjustment in the presence of discrepant data. Ser Adv Math Appl Sci 2004;66:37-51.

[3] Takamasu K, Furutani R, Ozono S. Basic concept of feature-based metrology. Measurement 1999;26:151-6.

[4] Sprauel JM, Linares JM, Bourdet P. Contribution of nonlinear optimization to the determination of measurement uncertainties. In: Selected Conference Papers of the 7th CIRP Seminar on Computer-Aided Tolerancing. 2001. p. 235-44

[5] Antunes SD, Vicente MAF. Estimation of the precision and uncertainty of a calibration. Ser Adv Math Appl Sci 2004;66:01-15.

[6] Weckenmann A, Lorz J, Beetz S. Monitoring coordinates measuring machine by user-defined calibrated parts. In: Proceeding of the 9th CIRP seminar on computer aided tolerancing, Tempe, USA; 2005. CD-Rom.

[7] Schwenke H, Siebert BRL, Waldele F, Kunzmann H. Assessment of uncertainties in dimensional metrology by Monte Carlo simulation. Proposal of a modular and visual software. Ann CIRP 2000;49:395-8.

[8] Balsamo A, Di Ciommo M, Mugno R, Rebaglia BI, Ricci E, Grella R. Evaluation of CMM uncertainty though Monte Carlo simulations. Ann CIRP 1999;48:429-32.

[9] Sommer KD, Siebert BRL, Kochsiek M, Weckenmann A. A systematic approach to the modelling and uncertainty analysis of measurement. In: Proceeding of the 12th International Metrology Congress; 2005. CD-Rom.

[10] Kurfess T, Banks DL. Statistical verification of conformance to geometric tolerance. Comput Aid Des 1995;27:353-61.

[11] Choi W, Kurfess T. Uncertainty of extreme fit evaluation for three-dimensional measurement data analysis. Comput Aid Des 1998;30:549-57.

[12] Yau HT. Evaluation and uncertainty analysis of vectorial tolerances. Precision Eng 1997;20: 123-37.

[13] Summerhays KD, Henke RP, Balwin JM, Cassou JM, Brown CW. Optimizing discrete point sample patterns and measurement data analysis on internal cylindrical surfaces with systematic form deviations. Precision Eng 2002;26: 105-21

[14] Henke RP, Summerhays KD, Balwin JM, Cassou JM, Brown CW. Methods for evaluation of systematic geometric deviations in machined parts and their relationships to process variables. Precision Eng 1999;23:273-92.

[15] Phillips SD, Borchardt B, Estler WT, Buttress J. The estimation of mea ment uncertainty of small circular features measured by coordinate measu machines. Precision Eng 1998;22:87-97.

[16] Sprauel JM, Linares JM, Bachmann J, Bourdet P. Uncertainties in CMM Measurements, Control of ISO Specifications. Ann CIRP 2003;52:423-6.

[17] Aranda S, Linares JM, Sprauel JM. A study of the likelihood function maximization against the signature shape. In: Proceeding of the 10th CIRP CAT Seminar Erlangen, Germany; 2007. CD-Rom.

[18] Eslter WT. Measurement as inference: fundamental ideas. Ann CIRP $1999 ; 48(2): 611-32$. 